\title{
Vero Cells as a Model to Study the Effects of Adenoviral Gene Delivery Vectors on the RNAi System in Context of Viral Infection
}

\author{
Alexey A. Matskevich ${ }^{\mathrm{a}}$ Jiun-Shan Jung ${ }^{\mathrm{a}}$ Michael Schümann ${ }^{\mathrm{b}}$ Manel Cascalloc \\ Karin Moelling a \\ a Institute of Medical Virology, University of Zurich, Zurich, Switzerland; b Institute of Virology, Philipps University \\ Marburg, Marburg, Germany; and 'Institut Català d'Oncologia, L'Hospitalet de Llobregat, Barcelona, Spain
}

\section{Key Words}

Vero cells $\cdot$ Interferon $\cdot$ RNAi $\cdot$ Viral infections

\begin{abstract}
Technology based on RNA interference (RNAi) is a promising source for new antiviral therapies. Although the application of RNAi has been studied extensively, significant problems with using RNAi remain. Very few studies have specifically assessed model systems for testing the effects of viruses or gene delivery vectors on the RNAi system. Since viruses have developed efficient strategies to circumvent the interferon (IFN) response, an IFN-deficient model system should be considered. Here we show that in Vero cells, which lack IFN$\alpha$ and IFN- $\beta$ genes, knockdown of Dicer, a key RNAi component, led to accelerated death of cells infected with other evolutionary distinct viruses: influenza A virus, vesicular stomatitis virus and poliovirus. We also demonstrate that transduction of Vero cells with adenoviral vector with subsequent infection with influenza $A$ virus also resulted in increased mortality of infected cells. These effects were much weaker in IFN-producing A549 and Hela cell lines. Thus, the Vero cell line could serve as an interesting model for studying the effects of gene delivery vectors on the RNAi system in the context of virus-related disorders.
\end{abstract}

RNA interference (RNAi) is a powerful gene knockdown technique that allows specific suppression of gene expression. Initial studies on the application of RNAi as an antiviral therapy have been encouraging. Preliminary trials using a mouse model have suggested that RNAi may be useful for the treatment of various viral diseases [1]. Although substantial progress is being made, significant problems with using RNAi remain.

These problems include toxicity and immune and inflammatory responses, which are caused by the overexpression of small interfering RNAs (siRNAs) or small hairpin RNAs (shRNAs) introduced by viral vectors. It has been reported that gene delivery vectors expressing shRNAs transduced into human tissues may activate immune mechanisms, in particular the interferon (IFN) system [2]. The enhanced response of the immune system to viral vectors makes it difficult for repetitive gene therapy in patients. Grimm et al. [3] showed that mice died from liver toxicity after being injected with high doses of shRNA designed against hepatitis B virus. The original promoter sequence used for driving production of shRNA created large amounts of RNA that saturated the RNAi system [3]. Studies performed by other groups have shown that high levels of shRNAs are toxic in tissue culture cells, and toxicity of shRNA directed against CCR5 for anti-HIV therapy has also been reported $[4,5]$.

\section{KARGER}

Fax +4161306 1234 E-Mail karger@karger.ch www.karger.com
Dr. Alexey A. Matskevich

Mucosal Immunity Laboratory, Department of Gynecology

Obstetrics and Medical Genetics, Central University Hospital of Vaud (CHUV)

Av. Pierre Decker 2, CH-1011 Lausanne (Switzerland)

Tel. +41 21314 3169, Fax +41 21314 4471, E-Mail amatskev@chuv.ch 


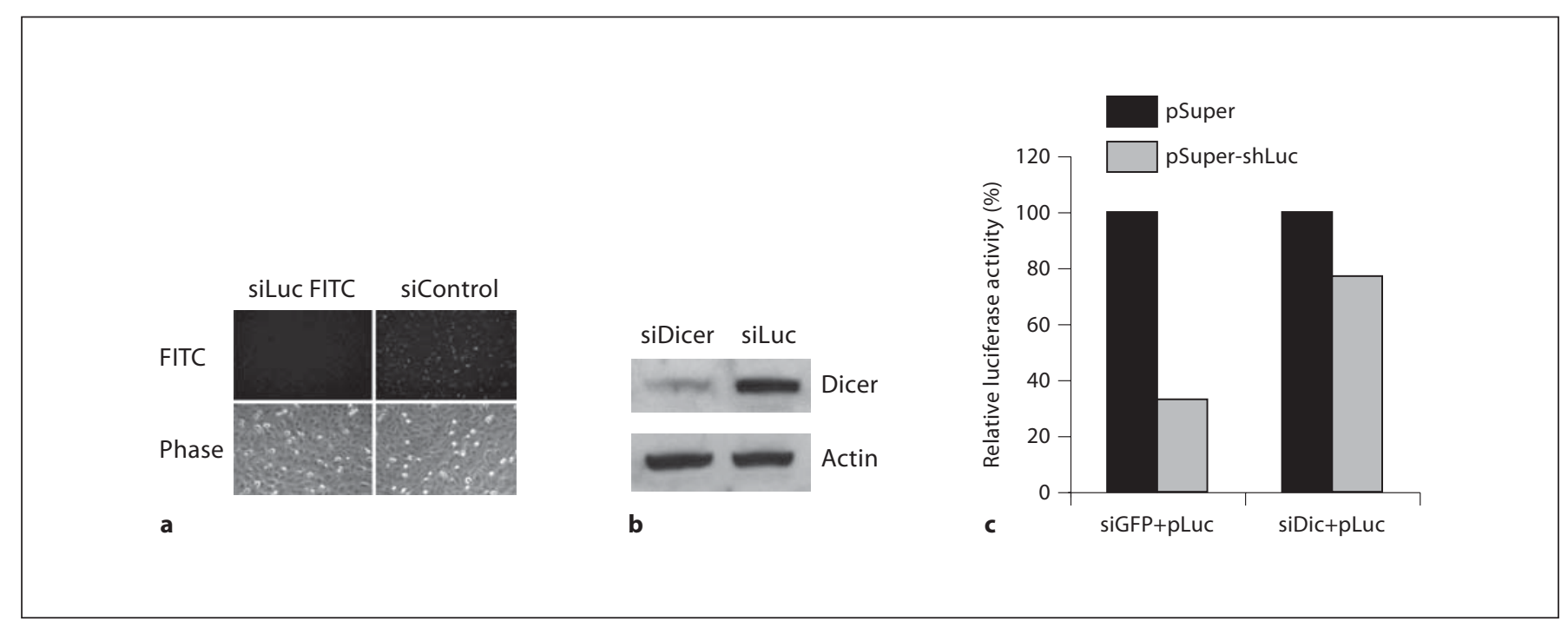

Fig. 1. Knockdown of Dicer in Vero cells. a Efficiency of transfection in Vero cells. Cells were transfected with siRNAs against luciferase and control FITC-conjugated control siRNA, and analyzed by fluorescent microscopy $18 \mathrm{~h}$ after transfection. b Efficiency of down-regulation of Dicer. Cells were transfected with siRNAs against luciferase or Dicer. $48 \mathrm{~h}$ later, proteins were extracted from transfected cells and Western blotting was per-

Why and how RNAi causes toxicity is not fully understood. One proposal is that the exogenously added shRNAs may compete with endogenous microRNAs (miRNAs) for the same cellular machinery that allows the RNAi-mediated silencing process to take place $[5,6]$. Additionally, RNAi has been proposed to be involved in the response to viral infection in vertebrates $[7,8]$. Indeed, cellular miRNA restricts the replication of the primate foamy virus-1 in human cells [9]. It has also been shown that Dicer, which is responsible for miRNA processing, inhibited HIV-1 replication in peripheral blood mononuclear cells [10]. Furthermore, RNAi and the IFN system may cross-react, and excessive exploitation of RNAi may affect the whole defensive system [11-13]. It has been shown, for example, that adenoviral virus-associated (VA) RNA I prevents the activation of protein kinase R and at the same time acts as a suppressor of RNAi $[14,15]$. Thus, model systems for testing the effects of viruses or gene delivery vectors on the RNAi system should be considered.

We have recently shown that in IFN-deficient Vero cells the knockdown of Dicer, a key component of the RNAi machinery, leads to accelerated cell death upon infection with influenza $A$ virus and to an increase of virus production. These effects were much weaker in the IFN- formed. c Knockdown of Dicer decreases inhibitory activity of RNAi. Cells were transfected with siRNAs against GFP or Dicer, and $36 \mathrm{~h}$ later they were cotransfected with plasmid expressing luciferase (pLuc) and plasmid expressing short hairpin RNA against luciferase (pSuper-shLuc). Plasmid pSuper was used as a control. Luciferase activity was measured $48 \mathrm{~h}$ after second transfection.

producing alveolar epithelial A549 cell line [11]. These results suggested that the involvement of RNAi in the antiviral response is overshadowed by the IFN system. Importantly, viruses have developed strategies to evade the IFN response, either by limiting IFN production or by blocking IFN actions [16]. Thus, Vero cells may provide valuable information on the role of RNAi in the complete absence of IFN.

In this study, we first tested whether Dicer is involved in the antiviral response of Vero cells infected with other evolutionarily distinct viruses. In doing so, we knocked down Dicer by transfecting the cells with siRNAs against it [17]. siRNA against luciferase [18] - siLuc - served as a negative control. Transfection efficiency in Vero cells was around $80 \%$ (fig. 1a). We could achieve $70-80 \%$ downregulation of Dicer, as measured by protein level (fig. 1b). Functional characterization of transfected cells using a reporter assay revealed that the knockdown of Dicer disrupts RNA silencing (fig. 1c). As the next step, the transfected Vero cells were infected with vesicular stomatitis virus (VSV), poliovirus type 1 and influenza A virus. In all the cases, knockdown of Dicer led to accelerated death of infected cells (fig. 2a-c). The infection of another IFNproducing cell line (Hela) with poliovirus showed no difference between Dicer knockdown and control siLuc 


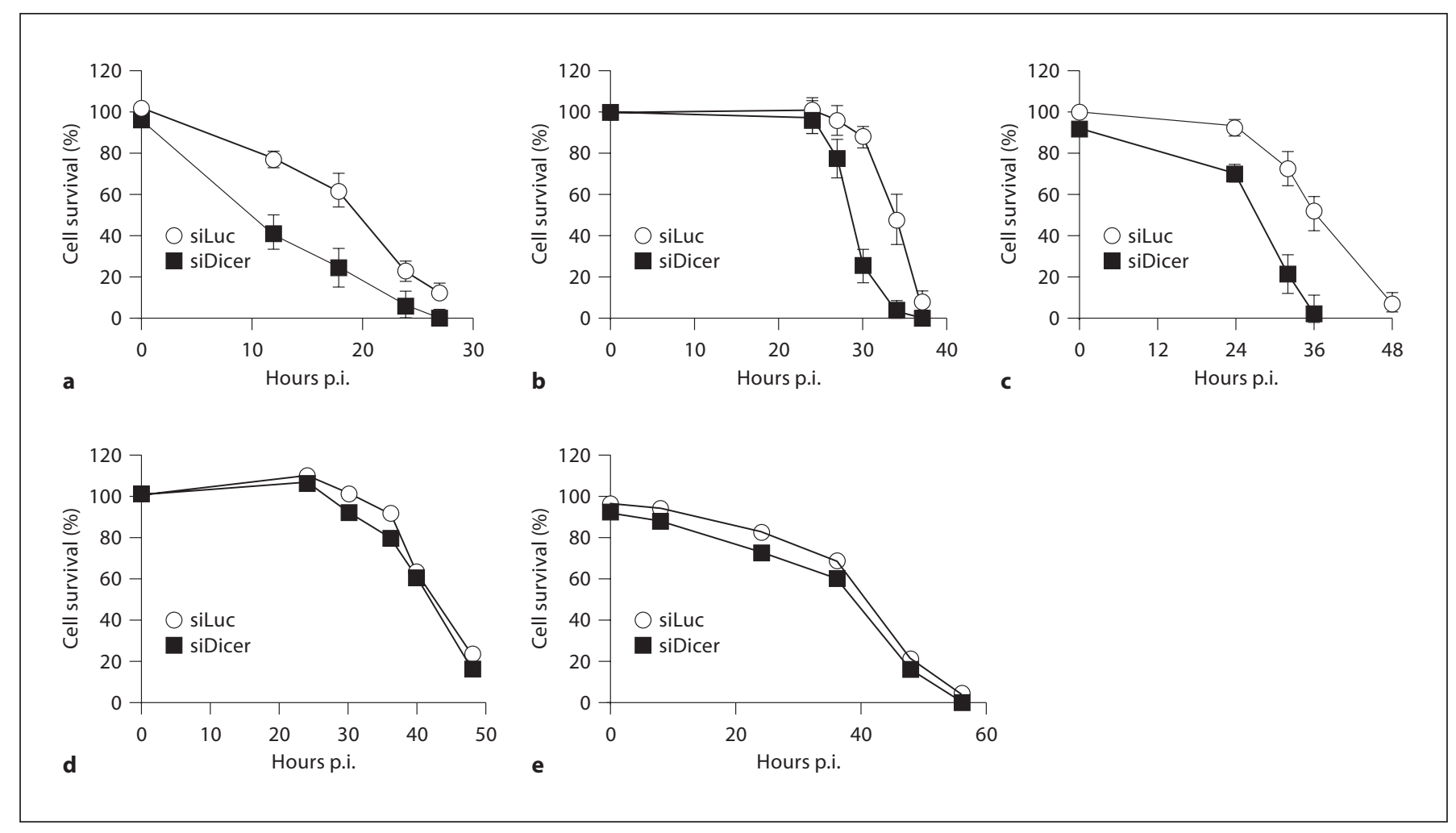

Fig. 2. Knockdown of Dicer affects survival of Vero cells after infection with different viruses. Cells were transfected with siRNAs against luciferase or Dicer, and $48 \mathrm{~h}$ later they were infected with VSV at MOI 0.05 (a), poliovirus at MOI 0.02 (b) and Influenza A virus at MOI 1 (c). Alive cells were counted. The survival rate of Hela cells infected with poliovirus at MOI 0.02 (d) and A549 cells infected with influenza A virus at MOI 1 (e) are shown for comparison. $\mathrm{p}<0.01$ for siDicer- versus siLuc-transfected cells at 12 and $18 \mathrm{~h}$ postinfection (p.i.; a), at 30 and $34 \mathrm{~h} \mathrm{(b),} \mathrm{and} \mathrm{at} \mathrm{24,} 32$ and $36 \mathrm{~h}$ (c), by Student's t test.

cells, which similar to the observation made for A549 cells infected with influenza A virus (fig. 2d, e). Thus, Dicer in Vero cells plays an important role not only in the case of influenza A virus, but also upon infection with other evolutionarily distinct viruses. Interestingly, hypersusceptibility to VSV infection due to impaired miR24 and miR93 expression has been previously shown in $\mathrm{Di}$ cerl-deficient mice [19].

The results of the experiments described above demonstrate that the downregulation of Dicer makes Vero cells very sensitive to infection with different viruses. Interestingly, some RNAi-based gene delivery viral vectors may also affect and, in some cases, even saturate the RNA silencing pathway $[3,20]$. It has been shown that adenoviral VA RNAs I and II may act as RNAi suppressors [15]. Adenoviral RNAs can inhibit Dicer function and can be processed to functional viral miRNAs that could help to saturate the silencing machinery $[21,22]$. Wild-type ad- enovirus infection has been shown to inactivate the RNA induced silencing complex in tissue culture [15]. Also in our experiments, adenovirus caused accelerated cell death and the effect was weaker when a mutant VA RNAdeficient adenovirus [23] was used (fig. 3a).

Thus, to assess the effects of adenoviral vectors on the RNAi system, we transduced Vero cells and control A549 cells with an adenoviral vector expressing green fluorescent protein (rAd-GFP) at equal MOI and subsequently infected the cells with influenza A virus. We used influenza A virus infection, since it has been characterized previously [11]. In control A549 cells the expression of GFP was lower (results not shown) and transduction had limited effects on cell survival (fig. 3b), possibly due to a complex reaction of the IFN system [24, 25]. However, in Vero cells the effects were much stronger (fig. 3c), suggesting that Vero cells are more susceptible to treatment with adenoviral vectors. Importantly, Vero cells trans- 


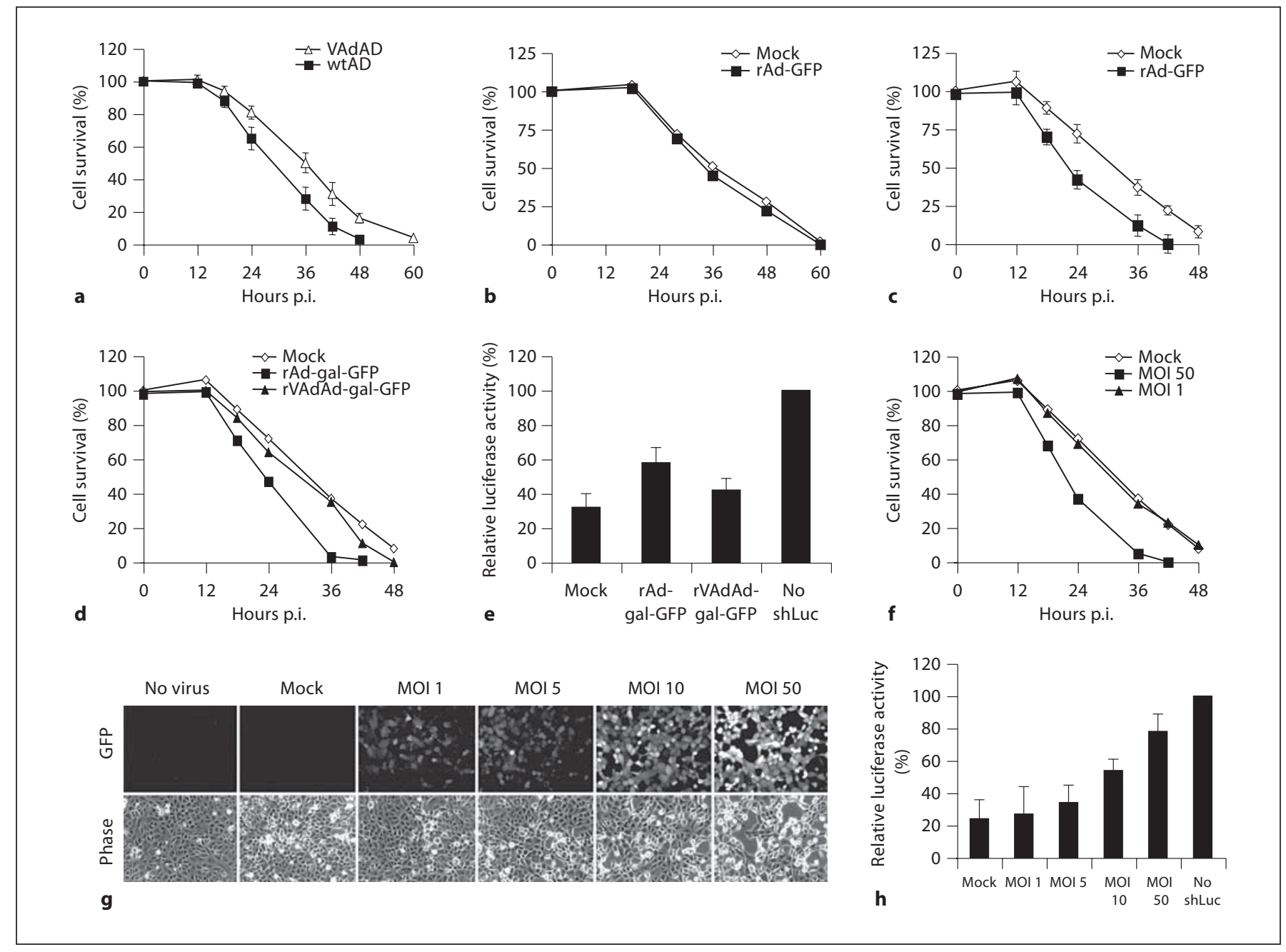

Fig. 3. Transduction of Vero cells with adenoviral vector. a Vero cells were infected with wild-type adenovirus (wtAD) or VA RNA-deficient adenovirus (VAdAD) at MOI 10, and alive cells were counted. $\mathrm{p}<0.05$ for VAdAD versus wtAD at 24,36 and $40 \mathrm{~h}$ postinfection (p.i.) by Student's t test. Control A549 (b) or Vero cells (c) were transduced with adenoviral vector expressing GFP (rAd-GFP) at MOI 10. Five days later they were infected with influenza A virus and alive cells were counted. $p<0.01$ for rAdGFP versus mock-treated cells at 16, 24 and 36 h p.i. (c), by Student's t test. d Vero cells were transduced with wild-type (rAd-galGFP) or VA RNA-deficient adenoviral vectors (rVAdAd-gal-GFP) at MOI 10. Three days later they were infected with influenza A virus and alive cells were counted. e Vero cells were transduced similar to (d) and 3 days later cotransfected with plasmid express- ing luciferase and plasmid expressing short hairpin RNA against luciferase. Luciferase activity was measured $36 \mathrm{~h}$ after transfection. $\mathrm{p}<0.05$ for rAd-gal-GFP versus mock-treated cells, by Student's t test. $\mathbf{f}$ Transduction of Vero cells at low MOI reduces the toxic effects of rAd-GFP. Vero cells were transduced with rAdGFP at different MOI, 5 days later they were infected with influenza A virus and the alive cells were counted (f) or analyzed by fluorescent microscopy 18 h postinfection (p.i.; g). $\mathbf{h}$ Vero cells were transduced with rAd-GFP, similar to (f) and 5 days later cotransfected with plasmid expressing luciferase and plasmid expressing short hairpin RNA against luciferase. Luciferase activity was measured $36 \mathrm{~h}$ after transfection. $\mathrm{p}<0.05$ for rAd-GFP at MOI 10 and MOI 50 versus mock-treated cells, by Student's $t$ test.

tor is less toxic (fig. 3d). This effect correlated with improved RNAi activity (fig. 3e). Further analysis showed that transduction of Vero cells with rAd-GFP at lower MOI significantly reduced the death of infected cells (fig. 3f, g). Again, these effects correlated with improved 
RNAi activity at low MOI, as shown by luciferase reporter assay (fig. 3h), whereas at high MOI RNAi activity was reduced to a level similar to that seen with artificial knockdown of Dicer, a key component of the RNAi pathway, (fig. 1c). Importantly, analysis of noninfected A549 or Vero cells showed that transduction with rAd-GFP did not significantly affect the morphology or growth rate of the cells during the course of experiments. However, the reduced growth of Vero cells transduced at high MOI was observed 10 days after transduction (results not shown). Comparable results were obtained when Vero cells were infected with poliovirus (results not shown). Interestingly, similar observations were made by Kay and colleagues [3] and Chen and colleagues [4], who showed that decreased expression of siRNAs reduced the toxic effects. Nontoxic doses of adenoviral vectors expressing shRNAs could be used therapeutically, and adenoviral shRNAmediated inhibition of expression of multidrug resistance protein 2 in a mouse model without detectable alteration of the cellular RNAi machinery was reported [27].

Importantly, transduction of Vero at MOI 1 and A549 cells at MOI 20 of rAd-GFP, which gave similar GFP expression levels, did not lead to RNAi suppression and thus resulted in similar survival rates after infection with influenza A virus (results not shown).

Thus, optimized expression of shRNAs will provide a powerful tool for efficient and yet therapeutically safe viral gene suppression. Taken together, these results suggest that adenoviral vectors may affect RNAi and that Vero cells could serve as a model to study the effects of such vectors on the RNAi system. Further studies are required to determine whether RNAi is directly involved in the antiviral response, as has been suggested for primate foamy virus, VSV and HIV $[9,10,19]$, or whether adenovirus-mediated inactivation of RNAi leads to a loss of miRNAs which are involved in the control of other defensive systems and thus make cells more susceptible to infections. However, we cannot exclude the possibility that the higher susceptibility of Vero cells to adenovirus transduction compared to A549 cells and the increased toxicity of wild-type adenovirus compared to VA RNAdeleted virus may occur as a result of reasons unrelated to the RNAi and IFN system. Vero cells could express more viral receptors or allow a more effective internalization or nuclear transport of viral particles. This suggests that other cell lines could be used to evaluate the toxicity of adenoviral vectors when the proper MOI is employed. Other viral vectors can be tested too.

In the present study, we focused on survival, which is one of the most important and informative parameters of the susceptibility of Vero cells to infection. However, similar investigations could be performed using more specific readouts, such as apoptosis or activation of different pathways. The advantage of Vero cells is that these cells are well characterized, can accommodate a large number of different viruses and are easier to handle than primary cells. Vero cells can be infected with SV-40, measles, arboviruses, reoviruses, rubella, simian adenoviruses, polioviruses, influenza viruses, respiratory syncytial viruses, vaccinia, and others [28-30]. Vero cells are used for many purposes, including screening and testing of bacterial toxins and pharmaceutical compounds, for the preparation of influenza virus vaccines and diagnostic reagents. Furthermore, Vero cells can potentially remove the necessity to generate specific cell lines and transgenic mice, which is costly and ethically problematic. Although used routinely in laboratory practice, other IFNproducing cells have limitations as a host system, since the effects of IFN may overshadow the contribution of other IFN-independent systems and RNAi [11].

In summary, our results indicate that the Vero cell line could serve as an interesting model for studying the effects of gene delivery vectors on the RNAi system in the context of virus-related disorders.

\section{Acknowledgements}

We thank Dr. Silvio Hemmi for providing the rAd-GFP viral vector and Anne Hempel for the initial studies performed with poliovirus.

\section{References}

\footnotetext{
Kim DH, Rossi JJ: Strategies for silencing human disease using RNA interference. Nat Rev Genet 2007;8:173-184.

-2 Bridge AJ, Pebernard S, Ducraux A, Nicoulaz AL, Iggo R: Induction of an interferon response by RNAi vectors in mammalian cells. Nat Genet 2003;34:263-264.

- 3 Grimm D, Streetz KL, Jopling CL, Storm TA, Pandey K, Davis CR, Marion P, Salazar F, Kay MA: Fatality in mice due to oversaturation of cellular microRNA/short hairpin RNA pathways. Nature 2006;441:537-541.

4 An DS, Qin XF, Auyeung VC, Mao SH, Kung SK, Baltimore D, Chen IS: Optimization and functional effects of stable short hairpin RNA expression in primary human lymphocytes via lentiviral vectors. Mol Ther 2006; 14:494-504.

5 Frantz S: Safety concerns raised over RNA interference. Nat Rev Drug Discov 2006;5: 528-529.
} 
-6 Castanotto D, Sakurai K, Lingeman R, Li H, Shively L, Aagaard L, Soifer H, Gatignol A, Riggs A, Rossi JJ: Combinatorial delivery of small interfering RNAs reduces RNAi efficacy by selective incorporation into RISC. Nucleic Acids Res 2007;35:5154-5164.

7 Bennasser Y, Yeung ML, Jeang KT: HIV-1 TAR RNA subverts RNA interference in transfected cells through sequestration of TAR RNA-binding protein, TRBP. J Biol Chem 2006;281:27674-27678.

$\checkmark 8$ Gatignol A, Lainé S, Clerzius G: Dual role of TRBP in HIV replication and RNA interference: viral diversion of a cellular pathway or evasion from antiviral immunity? Retrovirology 2005;2:65-71.

$\checkmark 9$ Lecellier CH, Dunoyer P, Arar K, LehmannChe J, Eyquem S, Himber C, Saib A, Voinnet O: A cellular microRNA mediates antiviral defense in human cells. Science 2005;308: 557-560.

10 Triboulet R, Mari B, Lin YL, et al: Suppression of microRNA-silencing pathway by HIV-1 during virus replication. Science 2007;315:1579-1582.

11 Matskevich AA, Moelling K: Dicer is involved in protection against influenza A virus infection. J Gen Virol 2007;88:26272635.

-12 O'Connell RM, Taganov KD, Boldin MP, Cheng G, Baltimore D: MicroRNA-155 is induced during the macrophage inflammatory response. Proc Natl Acad Sci USA 2007;104: 1604-1609.

13 Saumet A, Lecellier CH: Anti-viral RNA silencing: do we look like plants? Retrovirology $2006 ; 3: 3-8$.
4 Kitajewski J, Schneider RJ, Safer B, Munemitsu SM, Samuel CE, Thimmappaya B, Shenk T: Adenovirus VAI RNA antagonizes the antiviral action of interferon by preventing activation of the interferon-induced eIF2 alpha kinase. Cell 1986;45:195-200.

15 Andersson MG, Haasnoot PC, Xu N, Berenjian S, Berkhout B, Akusjarvi G: Suppression of RNA interference by adenovirus virus associated RNA. J Virol 2005;79:9556-9565.

-16 Goodbourn S, Didcock L, Randall RE: Interferons: cell signalling, immune modulation, antiviral response and virus countermeasures. J Gen Virol 2000;81:2341-2364.

17 Hutvagner G, McLachlan J, Pasquinelli AE, Balint E, Tuschl T, Zamore PD: A cellular function for the RNA-interference enzyme Dicer in the maturation of the let- 7 small temporal RNA. Science 2001;293:811-813.

$>18$ Chendrimada TP, Gregory RI, Kumaraswamy E, Norman J, Cooch N, Nishikura K, Shiekhattar R: TRBP recruits the Dicer complex to Ago2 for microRNA processing and gene silencing. Nature 2005;436:740-744.

19 Otsuka M, Jing Q, Georgel P, New L, Chen J, Mols J, Kang YJ, Jiang Z, Du X, Cook R, Das SC, Pattnaik AK, Beutler B, Han J: Hypersusceptibility to vesicular stomatitis virus infection in Dicer1-deficient mice is due to impaired miR24 and miR93 expression. Immunity 2007;27:123-134.

20 Snove O Jr, Rossi JJ: Expressing short hairpin RNAs in vivo. Nat Methods 2006;3:689695.

-21 Aparicio O, Razquin N, Zaratiegui M, Narvaiza I, Fortes P: Adenovirus virus-associated RNA is processed to functional interfering RNAs involved in virus production. J Virol 2006;80:1376-1384.

-22 Lu S, Cullen BR: Adenovirus VA1 noncoding RNA can inhibit small interfering RNA and microRNA biogenesis. J Virol 2004;78: 12868-12876.
23 Cascallo M, Gros A, Bayo N, Serrano T, Capella G, Alemany R: Deletion of VAI and VAII RNA genes in the design of oncolytic adenoviruses. Hum Gene Ther 2006;17:929940.

24 Flaherty DM, Hinde SL, Monick MM, Powers LS, Bradford MA, Yarovinsky T, Hunninghake GW: Adenovirus vectors activate survival pathways in lung epithelial cells. Am J Physiol Lung Cell Mol Physiol 2004; 287:L393-L401.

25 Weber F, Wagner V, Kessler N, Haller O: Induction of interferon synthesis by the PKRinhibitory VA RNAs of adenoviruses. J Interferon Cytokine Res 2006;26:1-7.

26 Schümann M, Dobbelstein M: Activating Ras mutations fail to ensure efficient replication of adenovirus mutants lacking VARNA. Cell Cycle 2006;5:315-321.

27 Narvaiza I, Aparicio O, Vera M, Razquin N, Bortolanza S, Prieto J, Fortes P: Effect of adenovirus-mediated RNA Interference on endogenous microRNAs in a mouse model of multidrug resistance protein 2 gene silencing. J Virol 2006;80:12236-12247.

28 Montagnon BJ: Polio and rabies vaccines produced in continuous cell lines: a reality for Vero cell line. Dev Biol Stand 1989;70: 27-47.

29 Montagnon BJ, Vincent-Falquet JC: Experience with the Vero cell line. Dev Biol Stand 1998;93:119-123

30 Yoshumura Y: Establishment of kidney cell strain of Cercopithecus aethiops. 14th Japan Conference of Tissue Culture Association, 1962. 